\title{
Correction to: CORM-2-Solid Lipid Nanoparticles Maintain Integrity of Blood-Spinal Cord Barrier After Spinal Cord Injury in Rats
}

\author{
Hari Prasad Joshi ${ }^{1} \cdot$ Hemant Kumar $^{2}$ • Un Yong Choi ${ }^{1}$ - Yong Cheol Lim ${ }^{3} \cdot$ Hyemin Choi $^{1} \cdot$ Juri Kim ${ }^{1} \cdot$ Jae Won Kyung ${ }^{1}$. \\ Seil Sohn ${ }^{1} \cdot$ Kyoung-Tae Kim ${ }^{4,5} \cdot$ Jin-Ki Kim $^{6} \cdot$ Inbo Han $^{1}$
}

Published online: 8 December 2020

(C) Springer Science+Business Media, LLC, part of Springer Nature 2020

\section{Correction to: Mol Neurobiol (2020) 57:2671-2689.} https://doi.org/10.1007/s12035-020-01914-5

The original version of this article unfortunately contained some mistakes.

There are changes in the order of authorship.

Un Yong Choi is not co-first author.

In-Bo Han should be written as Inbo Han.

Publisher's Note Springer Nature remains neutral with regard to jurisdictional claims in published maps and institutional affiliations.

The online version of the original article can be found at https://doi.org/ 10.1007/s12035-020-01914-5

Inbo Han

hanib@cha.ac.kr

1 Department of Neurosurgery, CHA University School of medicine, CHA Bundang Medical Center, 59 Yaptap-ro, Bundang-gu,

Seongnam-si 13496, Gyeonggi-do, Republic of Korea

2 Department of Pharmacology and Toxicology, National Institute of Pharmaceutical Education and Research (NIPER)-Ahmedabad, Gandhinagar, Gujarat, India

3 Department of Neurosurgery, Ajou University Hospital, Suwon, South Korea

4 Department of Neurosurgery, School of Medicine, Kyungpook National University, Daegu, South Korea

5 Department of Neurosurgery, Kyungpook National University Hospital, Daegu, South Korea

6 College of Pharmacy and Institute of Pharmaceutical Science and Technology, Hanyang University, Ansan 15588, Gyeonggi-do, Republic of Korea 